\title{
Photon Acceleration from Rest to the Speed of Light
}

\author{
C. H. Lai, T. Katsouleas, R. Liou, USC, Los Angeles, CA 90089 \\ W. B. Mori, C. Joshi, P. Muggli, R. Brogle, J. Dawson, UCLA, Los Angeles, CA 90024
}

\section{Abstract}

A new mechanism for generating coherent radiation from a laser-ionized gas-filled capacitor array is presented. This scheme directly converts a static electric field of wave number $\mathrm{k}_{0}$ into coherent radiation pulses. The physical mechanism is analogous to photon acceleration in plasma waves, and in this sense, photons are accelerated from zero frequency (zero energy) to a frequency $\omega_{\mathrm{p}}^{2} / 2 \mathrm{k}_{0} \mathrm{c}$. The photon frequency is tuned by gas pressure and/or capacitor spacing. In this paper we briefly describe the theory of this scheme and then present the preliminary experimental setup and results.

\section{INTRODUCTION}

Photon acceleration by using relativistic plasma waves has been investigated by Wilks, et al[1]. In this scheme, two short pulses of laser light spaced appropriate plasma wavelengths apart are injected into a plasma. Photons of the second light pulse then absorb energy from the plasma wake excited by the first laser pulse and are accelerated via their frequency upshift (frequency upshifting increases their group velocity in the plasma as well as their energy). It has also been found that the photon frequency can be significantly upshifted by a time-varying medium [24],e.g., by a relativistic ionization front rather than a plasma wave. The use of laser-produced ionization fronts have been successfully employed to upshift existing microwave radiation from $35 \mathrm{GHz}$ to over $170 \mathrm{GHz}$ in a plasma.[5].

In this paper, we describe another approach to generating radiation; namely from a static electric field by using a relativistic ionization front.[6]. From the viewpoint of acceleration, photons are accelerated from zero frequency, i.e., zero energy, to the frequency regime of microwaves to possibly IR. First, we analyze the radiation produced when an ionization front moves through a gas-filled capacitor array that is biased to produce a static electric field of wave number $k_{0}$ and zero frequency. The frequency and amplitude (output power) of the emitted radiation can be calculated by requiring the continuity of fields at the moving boundary. In the second part, we present he preliminary experimental setup and results.

\section{REVIEW OF THEORY}

\section{A. Frequency of the Radiation}

The geometry of this scheme is shown in Fig. 1. The alternately biased capacitors produce a static electric field of the form $\mathrm{E} \approx \mathrm{E}_{0} \sin \left(\mathrm{k}_{\mathrm{O}} \mathrm{x}\right) \hat{y}$ in a working gas of density $\mathrm{n}_{\mathrm{O}}$, where $\mathrm{k}_{\mathrm{O}}=\pi / \mathrm{d}$ and $\mathrm{d}$ is the spacing between adjacent capacitor plates. An ionization front (e.g., created by a short laser pulse) moves between the plates in the $+\hat{x}$ direction with a laser group velocity $\mathrm{v}_{f}=\mathrm{c}\left(1-\omega_{\mathrm{p}}{ }^{2} / \omega_{\mathrm{L}}{ }^{2}\right)^{1 / 2}$, where $\omega_{\mathrm{L}}$ is the laser frequency. To describe the radiation generated, we begin by considering the situation in a reference frame moving with the ionization front. In this frame, the Lorentz transformed electric field approximates an incident electromagnetic wave since the front velocity is close to the speed of light. The front is static in this frame and the incident wave gives rise to reflected and transmitted waves all at the same frequency. The reflected wave will be an extremely short pulse of hard x-rays with the amplitude approaching zero in the lab frame. The transmitted waves will turn out to be the tunable radiation that is the focus of this paper.

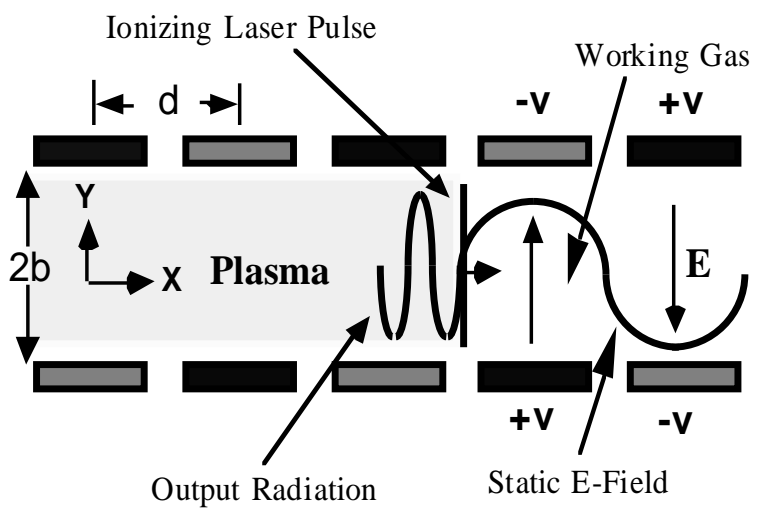

FIG.1 Schematic of a photon accelerator from rest to the speed of light

The frequency of the transmitted radiation can be easily obtained in the lab frame. The frequency follows two conditions: (i) the plasma dispersion relation, and (ii) continuity conditions at the front boundary. The dispersion relation is $\omega^{2}=\omega_{\mathrm{p}}{ }^{2}+\mathrm{c}^{2} \mathrm{k}^{2}$, while the equation for phase continuity is $\omega+\mathrm{kv}_{f}=\mathrm{k}_{0} \mathrm{v}_{f}$. When the front velocity is close to the speed of light (i.e., the laser frequency $\omega_{L}$ is much greater than the plasma frequency $\omega_{\mathrm{p}}$ ), the output frequency can be approximated as

$$
\omega \approx k_{0} v_{f} / 2+\omega_{p}^{2} / 2 k_{0} v_{f}
$$

It is instructive to construct graphical solutions to the above two equations[7]. The dispersion equation and continuous phase condition are plotted in Fig. 2; their 
intersection, marked by "T1", gives the output frequency and wavelength. Fig. 2 illustrates the case of $\mathrm{k}_{0}<\omega_{\mathrm{p}} / \mathrm{v}_{f}$ and we notice that the solution (T1 wave) has negative phase and group velocity which indicates that the output radiation moves in the same direction as the ionization front.

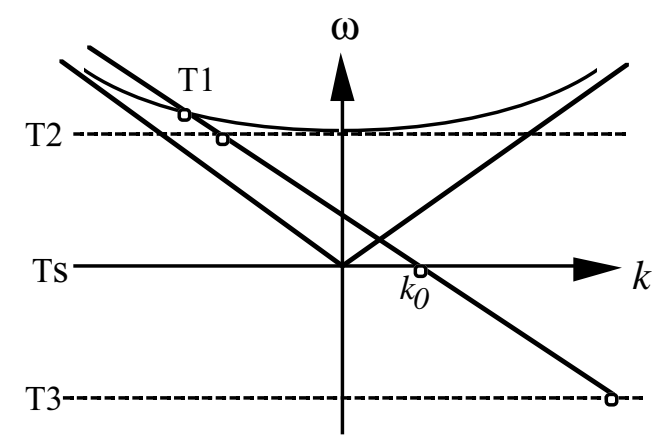

FIG.2 Dispersion diagram for electromagnetic, electrostatic, and free streaming plasma modes intersected by line of constant phase.

\section{B. Output Power of the Radiation}

The output power can be calculated by finding the transmission and reflection coefficients at the ionization front boundary. Besides the reflected wave and transmitted radiation, three more modes, i.e., two longitudinal modes and one free streaming mode, can also be excited[6]. They are also shown in Fig. 2, marked as "T2", "Ts", and "T3". The determination of these coefficients requires five boundary conditions. In addition to the usual conditions that (1) $E_{\text {tangential }}$ and (2) $B_{\text {tangential }}$ be continuous, three more conditions follow from the fact that electrons are 'born' at rest with no initial velocity at the moment they are ionized. As a result, the current density $j_{\mathrm{y}}$ and $j_{\mathrm{X}}=0$, and the surface charge density $\rho_{\mathrm{s}}=0$ at the front. The complete set of boundary conditions that follow from these and Faraday's and Gauss' laws are continuity of (1) $E_{y}$ (2) $B_{z}$ (3) $\partial B_{z} / \partial x+(1 / c) \partial E_{y} / \partial t(=0)(4) \partial B_{z} / \partial y-(1 / c) \partial E_{x} / \partial t$ $(=0)(5) E_{x}$. The details of this calculation have been shown in reference [6]. We simply state the results here: for relativistic fronts and large up-shifts, the coefficient of the T1 mode approaches one. That is, the output radiation amplitude is approximately equal to the amplitude of the static electric field.

\section{EXPERIMENTAL SETUP AND RESULTS}

\section{A. Setup}

An experiment was designed to verify photon acceleration from rest for output frequencies in the microwave range. Fig. 3 depicts the experimental block diagram. A pyrex glass tube and two stainless steel flanges form the vacuum chamber which was evacuated by a turbomolecular pump. Two quartz windows provide the vacuum interface for the laser and microwave radiation. Azulene vapor was chosen to be the working gas because it is easily ionized by a $266 \mathrm{~nm}$ laser[5]. In order to achieve sufficient azulene pressure, the experiment was operated at a temperature of $140^{\circ} \mathrm{C}$. At this elevated temperature the background pressure was abount 3 mTorr. The entire experimental setup was wrapped with heating tapes (not shown in the figure) to achieve the required temperature. A leak valve was used to control the amount of azulene vapor leaking into the chamber and therefore the gas pressure. A convectron gauge was used to monitor the pressure in the chamber. The structure consists of 12 pairs of copper electrodes (6 periods). The spacing between adjacent electrodes (d) was $4.7 \mathrm{~cm}$ with separation (2b) of $1.5 \mathrm{~cm}$. The structure was machined from high temperature plastic kel-F material. The material is mechanically stable and has high dielectric strength. The laser system produces an intense UV pulse $(266 \mathrm{~nm})$ with a duration of $50 \mathrm{ps}$ (FWHM) and total energy of $30 \mathrm{~mJ}$. The microwave radiation was detected with a combination of a horn antenna followed by a matched waveguide and a crystal detector in various bands. Three specific bands were used, namely, the X-band (with cut-off at $6.56 \mathrm{GHz}$ ), Ku-band (with cut-off at $9.49 \mathrm{GHz}$ ) and $\mathrm{K}$-band (with cut-off at $14.08 \mathrm{GHz}$ ). The structure was biased with a DC power supply to an operating voltage of $1 \mathrm{kV}$.

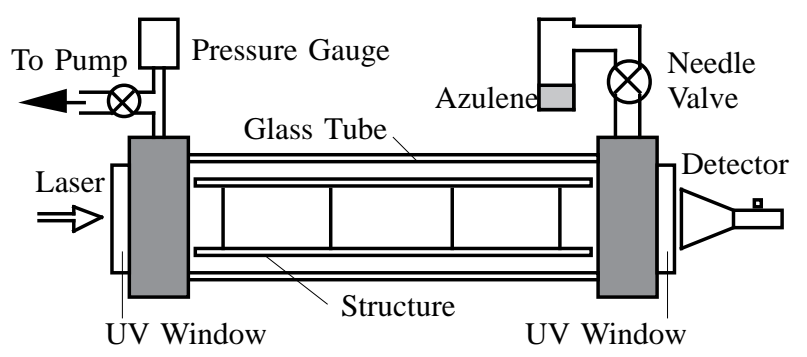

FIG.3 Experimental setup

\section{B. Preliminary results}

Null tests showed that the appearance of signals was directly associated with the presence of azulene, DC bias and the ionizing laser. The strength of signals detected on the crystal diode detector increased monotonically with the DC bias voltage and were typically in the range of $2-20 \mathrm{mV}$, depending on the sensitivity of crystal detectors. Based on cold tests we expect that the detected signal is 0.1 to $1 \%$ of the radiation generated within the device (primarily because of large impedance mismatches at the output of the vacuum structure). The signal was coincident with the 50ps laser signal and had a duration less than $5 \mathrm{~ns}$, the instrument limited time resolution of our electronics. The output was also polarized in the direction of the DC bias field typically with a 6 to 1 intensity ratio. 
Fig. 4 shows the amplitude of the detected signals measured with three different waveguides as a function of azulene pressure. The amplitudes of signals in this figure were normalized to the maximum signal obtained by the same set of waveguide and crystal detector. As the vapor pressure is increased, one can expected to achieve higher microwave frequencies. The dips occurs between 1.5 and 4 mTorr were thought to be due to the frequency characteristics, i.e., impedance mismatches, of this periodic structure.

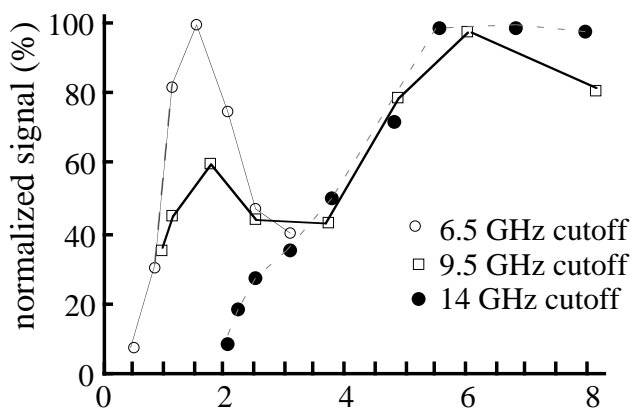

Azulene Pressure (mTorr)

FIG. 4 The normalized intensity of signals vs. azulene pressure

We also converted the azulene pressure to the corresponding plasma density based on a previous experiment done at UCLA with the same laser and similar controlled parameters [5]. The conversion factor was $9 \times 10^{11} \mathrm{~cm}^{-3}$ per $\mathrm{mT}$ of azulene. However, since the laser power in the present experiment was only $75 \%$ of the power used in the previous experiment, we expect the conversion factor to be $5.1 \times 10^{11} \mathrm{~cm}^{-3}$ per $\mathrm{mT}$ of azulene because the ionization rate is proportional to the square of laser power for such a two-photon ionization process. With this estimated conversion rate we were able to plot the frequencies of the minimum onset (i.e., the lowest points of the three curves in Fig. 4) vs. plasma density in Fig. 5. The solid line represents the theoretically predicted line given in Eq.(1). The data is in reasonable agreement with the theory.

\section{CONCLUSION}

We have described a new mechanism for generating coherent radiation by using an ionization front. This scheme is analogous to photon acceleration in relativistic plasma waves, and in this sense, the output radiation is directly converted from a static field of zero frequency. The output frequency is linearly proportional to the plasma density. In the proof-of-principle experiment we have observed wellpolarized signals coincident with the laser signal. We also plotted the relation between the output frequency and the plasma density by applying the conversion factor based on previous work. The preliminary results seem to to be in reasonable agreement with theoretical predictions. However, more accurate measurements on the plasma density and other related tests are necessary in order to further verify this mechanism.

Work supported by AFOSR Grant No. F49620-95-1-0248 and US DOE Grant No. DE-FG03-92ER-40745.

P. Muggli is supported by the "fonds national suisse de la recherche scientifique" grant number 8220-040122.

\section{REFERENCE}

1. S. C. Wilks, J. H. Dawson, W. B. Mori, T. Katsouleas and M. E. Jones, Phys. Rev. Lett. 62, 2600 (1989).

2. V. I. Semenova, sov. Radiophys. and Quantum electron. 10, 599 (1967).

3. M. Lampe, E. Ott and J. H. Walker, Phys. Fluids 10, 42 (1967).

4. W. B. Mori, Phys. Rev. A 44, 5118 (1991).

5. R. L. Savage, R. P. Brogle, W. B. Mori and C. Joshi, IEEE Trans. Plasma Sci. 21(1), 5(1993); R. L. Savage, W. B. Mori and C. Joshi, Phys. Rev. Lett. 68, 946(1992).

6. W. B. Mori, T. Katsouleas, J. M. Dawson, and C. H. Lai, Phys. Rev. Lett. 74, 542 (1995)

7. C. H. Lai, T.Katsouleas, W. B. Mori, D. Whittum, IEEE

Trans. Plasma Sci. 21(1), 45(1993).

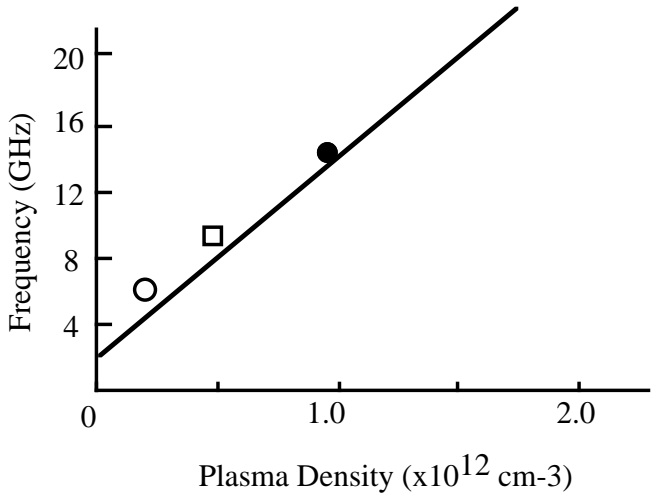

FIG. 5 Output frequency vs. plasma density. The solid line is the theoretical prediction. 